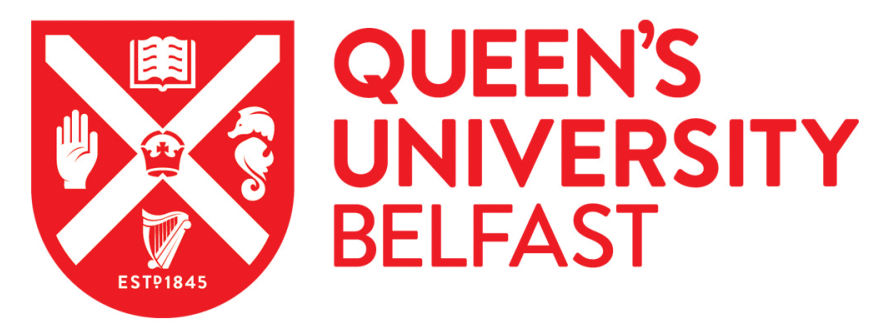

\title{
Enabling communities of practice surrounding the design and use of custom accessible music technology
}

Lucas, A., Schroeder, F., \& Ortiz, M. (2021). Enabling communities of practice surrounding the design and use of custom accessible music technology. Computer Music Journal, 44(2-3), 9-23.

https://doi.org/10.1162/COMJ_a_00567

Published in:

Computer Music Journal

Document Version:

Publisher's PDF, also known as Version of record

Queen's University Belfast - Research Portal:

Link to publication record in Queen's University Belfast Research Portal

Publisher rights

Copyright 2021 Massachusetts Institute of Technology..

This work is made available online in accordance with the publisher's policies. Please refer to any applicable terms of use of the publisher.

\section{General rights}

Copyright for the publications made accessible via the Queen's University Belfast Research Portal is retained by the author(s) and / or other copyright owners and it is a condition of accessing these publications that users recognise and abide by the legal requirements associated with these rights.

Take down policy

The Research Portal is Queen's institutional repository that provides access to Queen's research output. Every effort has been made to ensure that content in the Research Portal does not infringe any person's rights, or applicable UK laws. If you discover content in the Research Portal that you believe breaches copyright or violates any law, please contact openaccess@qub.ac.uk. 
Alex Lucas, Franziska Schroeder, and Miguel Ortiz

Sonic Arts Research Centre

Queen's University Belfast

4 Cloreen Park, Belfast, BT9 5HN UK

\{alucas02, f.schroeder, m.ortiz\}@qub.ac.uk
Enabling Communities of

Practice Surrounding the

Design and Use of Custom

Accessible Music

Technology

\begin{abstract}
In this article, the research group Performance without Barriers reflect on the process of collaboratively designing a custom guitar-inspired instrument with Eoin Fitzpatrick, a physically disabled musician from the Drake Music Project, Northern Ireland. As part of a longitudinal ethnographic case study designed to uncover factors that contribute to the longevity of custom assistive music technology, the authors monitored Fitzpatrick using this instrument over two months. The findings of this study inform a reflection on the social, technical, and environmental factors that the provision of such technology a reality. The authors make suggestions for ways to achieve long-term, sustained use. Custom technologies, seemingly unique on the surface, may well utilize similar underlying hardware and software components. Those involved in its design, fabrication, facilitation, and use could benefit from a concerted effort to share resources, knowledge, and skill as a mobilized community of practitioners. In such a pursuit, the authors recommend that practitioners consider strategies for managing the inherent complexity of digital technology. Fostering shared mental models within open-source communities can result in improved efficiency in the development of accessible music technology.
\end{abstract}

The commercial music technology sector is rife with tools to assist users in their creative pursuits. For instance, Deezer's Spleeter (https://github.com/deezer/spleeter) machinelearning library assists remix artists in music source separation, or Yousician (https://yousician.com), which helps musicians to learn their instruments by combining fast Fourier transform analysis with gamification techniques. Despite the increasing availability of technological tools that enable many to make and produce music, many people face disabling barriers in musicking, that is, participation, in any capacity in musical performance or composition (Small 1998). Access barriers can be overcome through the use of commercial accessible music technology (AMT) devices, such as Soundbeam (https://www.soundbeam.co.uk), Skoog (https://skoog.media), and The Beamz (http://thebeamz.com). As disability is often unique to an individual, custom technology holds great potential to address specific needs. For technology to be successful in removing access barriers, there is a requirement to consider specific social, technical,

Computer Music Journal, 44:2/3, pp. 9-23, Summer/Fall 2020 doi:10.1162/COMJ_a_00567

(c) 2021 Massachusetts Institute of Technology. and environmental factors. Unfortunately, it is often the case that custom AMT does not see sustained use (Samuels 2016). Device longevity is also problematic for new digital musical instruments (DMIs; cf. Morreale and McPherson 2017) and assistive technologies. Studies have found that users abandon up to 50 percent of assistive technologies within three years (Phillips and Zhao 1993). The authors' underlying assumption is that factors contributing toward the short life span of these two types of technology are likely to be compounded for custom AMT.

The Drake Music Project, Northern Ireland (DMNI) is one of several third-sector inclusive music organizations that use music technology to provide disabled people with access to musicking. Other prominent organizations include Share Music and Performing Arts, Heart $\mathrm{n}$ Soul, and the OneHanded Musical Instrument (OHMI) Trust. The first author of this article spent approximately three years working as an access music tutor with DMNI (2017-2020), and a further six months throughout 2020 with the OHMI Trust developing custom AMT. Based on his experience, this article explores critical factors affecting the adoption and longevity of custom AMT and suggests ways to achieve sustained use. 
Figure 1. Device One: USB

MIDI interface that

converts binary signals

from accessibility switches

to MIDI note messages.

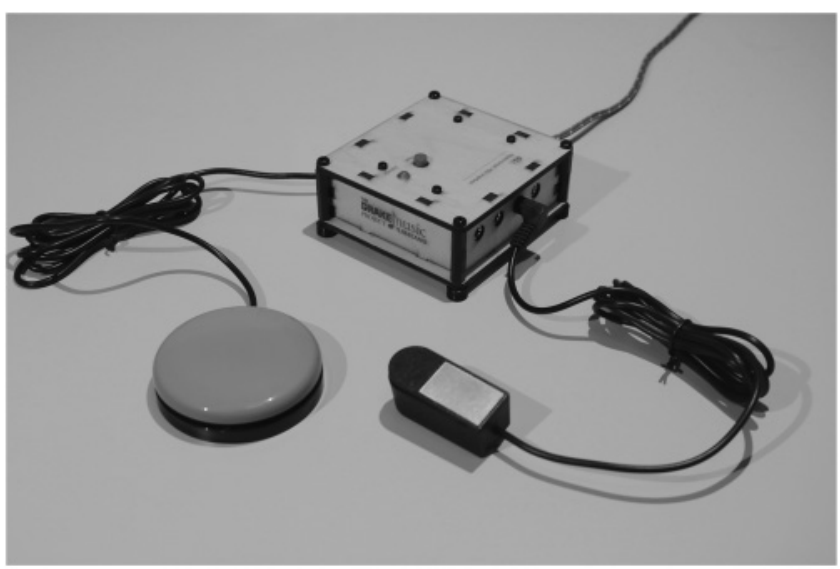

Terminology

The term assistive technology describes the technology designed to assist disabled people in various aspects of their daily life. Screen-reading software or computer access switches are examples of assistive technology. Some prefer the term "accessible technology," arguing that all technology can be said to assist users to some extent (Ladner 2010). Accessible digital musical instruments (ADMIs) is a term that is gaining popularity, especially within the New Interfaces for Musical Expression (NIME) community. A shortcoming of this term is the omission of accessible or enabling technological tools, used for musical purposes, which are not necessarily musical instruments in their own right. The Haptic Wave (Tanaka and Parkinson 2016) is an example of such technology, designed for audio engineers with visual impairments; it provides an interactive tactile representation of audio waveform amplitude.

Another example is Device One (https://github.com/alexmlucas/d1), a USB MIDI switch interface, pictured in Figure 1. Developed in a collaboration between DMNI and the first author, it allows control of music software by using computer access switches. To include technology of this kind, we have opted for the term accessible music technology. As a musician, composer, or engineer could operate such technology, in this article, the term "artist" is used to encompass all three.

Do-it-yourself assistive technology (DIY-AT), is a term used to describe custom, accessible technologies. Richard Phoenix (2020) highlights the privilege of being able to "do-it-yourself." In many situations, disabled people do not develop DIY-AT themselves. Phoenix notes that "Do-ItYourself can mean different things to different people and is a misleading term" (Phoenix 2020, point 4). Past research has offered "DIY-for-others" as alternative terminology (Parry-Hill et al. 2017). In being somewhat of a misnomer, however, we have opted for the term "custom AMT." Lastly, while custom AMT may be designed by or with an individual to remove specific access barriers, it may also prove to be enabling for others.

\section{Access Barriers to Musicking}

The Social Model of Disability (Withers 2012) describes disability as a condition that arises through the organization of society, the attitudes it holds, and the design of the environment in which it exists. A person is disabled by external factors, not by any particular impairment. A typical example of this phenomenon is a wheelchair user, disabled from entry to a public building through the omission of a mobility ramp. In this scenario, we can say that the person in question faces an access barrier. There are many potential access barriers to musicking, dependent on the specific task or activity the artist is aiming to achieve, the nature of the impairment and the environmental context. The complexities of overcoming access barriers become apparent when examining specific examples.

\section{The Piano and the Wheelchair}

In the context of musical performance, there is a requirement for musicians to have precise motor control. The piano in a traditional setting is a useful example. Disregarding its extraordinary inner workings, one might argue that the keyboard interface is conceptually simplistic, a series of keys each producing a different pitch when struck. Early interactions with a piano involve the novice player becoming proficient in targeting specific keys with specific fingers of one hand. Before long a second hand is 
Figure 2. Typical dimensions of an adult wheelchair alongside the dimensions of a Yamaha U3 acoustic piano, illustrating just one set of barriers between disabled musicians and traditional musical instruments.

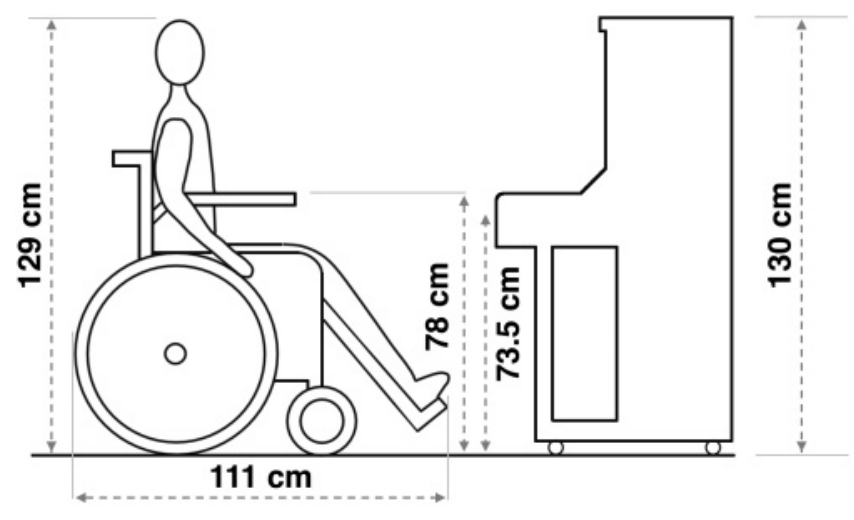

introduced. The player is soon using several fingers to press several keys simultaneously and rhythmically. The keyboard interface demands a set of affordances that may not be available to individuals with physical impairments-for example, cerebral palsy, a life-long condition affecting movement and coordination (NHS 2020).

The physical characteristics of the acoustic piano place requirements on the player. The keyboard mechanism is at a fixed height; it cannot move higher or lower. Convention dictates the player be seated on a specifically designed stool. The knees must be beneath the keyboard mechanism, feet in reach of the pedals and arms extended forward with fingers resting on the keys. Figure 2 depicts the average dimensions of an adult seated in a wheelchair (Hitchcock et al. 2006) alongside the approximate dimensions of a Yamaha U3 acoustic piano. On average, a wheelchair armrest is $78 \mathrm{~cm}$ from the floor. The top of the U3 keys are 73.5 $\mathrm{cm}$ from the floor; this may hinder the wheelchair user from getting suitably close to the piano keys. The comfortable reach of an adult wheelchair user is approximately $18 \mathrm{~cm}$ (DfT 2005). Although somewhat of a generalization, these factors could result in a wheelchair user playing the piano in an uncomfortable position or entirely prohibit the playing of the instrument.

How does an individual with an interest in playing and learning a musical instrument overcome disabling barriers such as the fixed dimensions of an acoustic piano? Part of the disabling barrier in this example lies in the design of the instrument and wheelchair technologies. These designs combined impose requirements on the musician. Theoretically, the barrier itself can be partially lifted through design. The critical challenge in overcoming access barriers lies not solely in conceptual design, but in its realization and application in a real-world context alongside a more inclusionary view of musicking. Ethnomusicologist and music-and-disability scholar Alex Lubet (2011) pointed to the fact that musical skill in Western societies is often judged by notions of accomplishment and virtuosity, excluding those deemed not good enough. His theory of social confluence views disability not as a fixed status but rather a fluid one based on sociocultural context.

\section{AMT Longevity in Northern Ireland}

The majority of custom AMT devices developed by DMNI are a result of short-term collaborative projects such as hackathons, involving disabled artists, access music tutors, and instrument makers. In 2015, in the first of these events, music technology students from Queen's University Belfast partnered with DMNI artists (Samuels and Schroeder 2019). The event, "Designing Inclusive Interactions," was designed with Matt Ratto's (2011) critical making methodology in mind. In this approach, materially productive engagement explored the gap between physical and conceptual ways of working. Over three days, custom AMT devices tailored toward the music access requirements of each artist were collaboratively designed and fabricated. Disabled artist Marylouise McCord co-designed a gestural instrument, where hand movements were captured and translated to sound using custom software and hardware.

Due to the success of the first design event, organizers planned a second edition for the following year. Recognizing the creative potential of the custom AMT co-designed a year prior, McCord decided that she would like to continue working on it. Unfortunately, the custom AMT no longer existed. With the hardware disassembled, and the software lost, to develop her instrument further, McCord would have had to start from scratch. Despite the technology used in AMT devices often 
being open source, in order for designs to be reused or adapted to suit an individual's unique needs, specialist expertise is required. In McCord's case, the technologist she had collaborated with was no longer working on the instrument nor in the institution. Unfortunately, there is a shortage of longitudinal studies into the broader use of custom AMTs (Frid 2019). As both assistive technology and new DMIs face issues with longevity (Phillips and Zhao 1993; Morreale and McPherson 2017), our assumption is the short life span of custom AMT is likely a pervasive issue, affecting inclusive music organizations more widely.

To help better understand factors that contribute toward the sustained use of custom AMT devices, we designed an ethnographic study. In similar fashion to past hackathons, a DMNI artist would codesign a custom AMT device. In this instance, the study would take place over six months, providing an opportunity to monitor longer-term goals. The study commenced in the autumn of 2018 and was divided into the following three stages, each stage being two months in duration:

1. Participatory Design: An iterative process, seeing prototypes fashioned, tested with the artist, and refactored.

2. Development: Technical work resulting in fabrication of a custom AMT device.

3. Usage: Direct observation and artist diaries monitoring the use of the custom AMT.

We discuss each stage in the following sections.

\section{Participatory Design}

There are two predominant frameworks used by occupational therapists in the design and evaluation of assistive technology: matching person and technology (MPT), and human activity assistive technology (HAAT).

\section{The Matching Person and Technology Model}

The MPT model takes three primary components into account: the person, the milieu, and the technology. There is an understanding that the first two components can have either a positive or a negative influence on the use of the technology. The technology may appear perfect for a specific need; but without the needed environmental support, the technology may go unused. The MPT model is expansive and thoroughly documented. A flowchart-based roadmap guides providers in the use of numerous forms and questionnaires. In brief, the process focuses initially on the person, detailing age, gender, lifestyle, goals, functional needs, and required adjustments. Other characteristics, such as the person's habits, upbringing, coping styles, social background, education, and profession, are also discussed. Next, the process focuses on the milieu, that is, the characteristics of the social environments of technology use, fostering an understanding of cultural, attitudinal, physical, economic, and legislative dependencies. This stage determines the frequency of exposure to the technology, the expectations from its use, and the social support available. Finally, the technology itself becomes the focus, taking into account its required performance, cost, comfort, availability, and appearance. The evaluation process culminates in the monitoring of the technology in use. (For further details, see https://sites.google.com/view/ matchingpersontechnology.)

\section{The Human Activity Assistive Technology Model}

The HAAT model dictates that for accessible technology to be effective, we must consider the following four interconnected facets:

1. Human: What are the goals, skills, and abilities of the artist?

2. Activity: What is the nature of the planned activity?

3. Technology: What features does it have, and how does one interact with it?

4. Context: What are the physical, social, and cultural attributes of the environment in which the three elements above exist?

For further details, see Cook and Livingston (2010) 
Figure 3. A mood board created by Eoin Fitzpatrick to illustrate his aesthetic tendencies and design goals for his custom instrument.

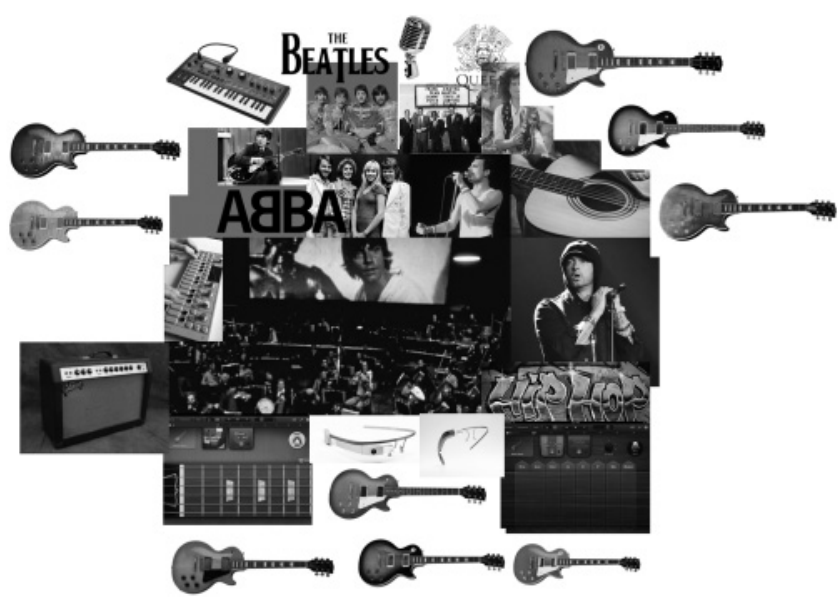

with his head tilted down, towards the left. He can move his head from this position around to the right by approximately 90 degrees. His mother fashioned a seamed cap featuring a frontal pocket into which an iPhone would sit. Via the app GyroSynth (https://www.beepstreet.com/ios/gyrosynth), positional data transmitted wirelessly as MIDI messages would trigger virtual instruments in Ableton Live.

\section{The Activity}

Fitzpatrick wanted to play electric rhythm guitar. To help the authors understand his goals, Fitzpatrick participated in initial design-thinking exercises, for example, the creation of a mood board, as depicted in Figure 3. The mood board helped identify Fitzpatrick's artistic goals and preferences, with the unintended positive consequence of serving as a communication aid. His goals were captured in user stories and use cases, a typical approach utilized by user experience designers to guide the design and subsequent development process. His user stories include the two following examples:

As a musician, I would like to perform with electric guitar sounds in a live environment. I must be in control of my instrument when performing, and it must be comfortable for me to play. 
Figure 4. Instrument One: A standalone virtual guitar instrument consisting of a base unit with internal speakers, $a$ wireless wristband with motion-tracking capabilities, and an access switch.
As a composer who works on new material regularly, I want my instrument to be relevant and applicable to the music I am composing. I want the instrument to integrate into my workflow.

\section{The Technology}

Fitzpatrick and the authors made an early decision to use digital technology to recreate the sound of the electric guitar, thereby reducing cost and potential mechanical complexity. The authors assumed that Fitzpatrick's left arm and head gestures would be the best way for him to play his instrument and therefore explored these interaction possibilities first. Inertial measurement unit (IMU) breakout boards, featuring six degrees of freedom, were attached to a 3-D printed headset and wristband he wore. We discovered that although Fitzpatrick can move his head across a range of 90 degrees, it is difficult for him to keep his head fixed once he has moved away from his natural resting position. Furthermore, he demonstrated a tendency to move his head back to its resting position when moving his arm. Alternative controllers were tested, such as a sip-and-puff switch, using an MPX5500DP pressure sensor (http://nxp.com). As Fitzpatrick would typically drink through a straw, sipping came very naturally to him. Puffing was challenging for him, being inclined to puff rapidly and to sip between puffs.

The authors explored ways of providing Fitzpatrick with visual feedback. An OLED screen was attached to the IMU headset and used to display chord symbols. Research suggests that disabled people have lower rates of literacy than nondisabled people (UNESCO 2018). We soon discovered we had incorrectly assumed that Fitzpatrick was literate, making this approach impracticable.

Although there were caveats in the use of the interface elements just described, it was necessary for us to remember that given the opportunity, through practice, Fitzpatrick had the potential to acquire new manual skills and knowledge. Over time, any dedicated musician may develop manual skills as an instrumentalist, and acquire musical knowledge; muscles can strengthen, sight-reading

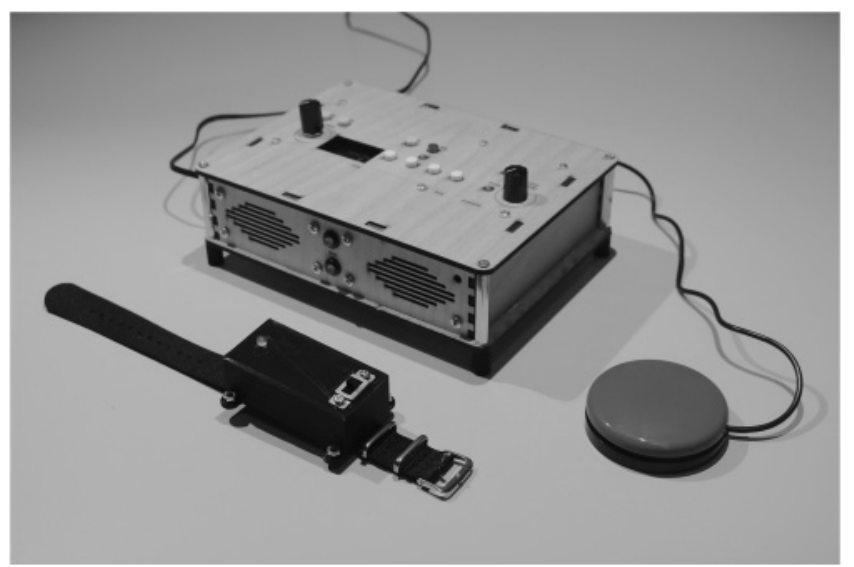

can improve. The same is true of Fitzpatrick and his custom guitar. Added to this, he is keen and committed; an excellent starting point for developing a custom instrument.

The design matured iteratively into a standalone device with internal speakers that became known as Instrument One, il (https://github.com/ alexmlucas/i1), as shown in Figure 4. Together, Fitzpatrick and the first author decided not to use an IMU to track head movement. Instead, the use of an off-the-shelf access switch was more accessible to Fitzpatrick. The switch is wheelchair mounted and positioned in reach of his chin, which he uses to select, in round-robin fashion, one of three preconfigured chords. The chord selection increments, each time the switch is pressed, before wrapping around from chord three to chord one. There is no way for him to reverse the direction of selection. With a palette of just three chords, this design limitation does not significantly impact the performative aspects of the instrument. A more extensive chord palette may, however, prove challenging to navigate in this manner. Visual feedback shows the selected chord through the use of multicolored LEDs. The authors found that Fitzpatrick can move his right arm across a broader range, and is more consistent in its movement in comparison to his left arm. A wristband on his right arm transmits positional pitch data wirelessly to the base unit of il, enabling him to pluck the strings of his virtual guitar. It is 
also possible for il to play backing tracks, loaded via a USB memory stick

\section{The Context}

Fitzpatrick was keen to be able to use his instrument not solely in DMNI music workshops but also at home, allowing him to practice his instrument and develop as an artist. His father, Peter, a "digital immigrant," is one of his son's primary carers. When the younger Fitzpatrick uses technology at home, for example, when listening to audiobooks via an MPEG-3 player, its use is facilitated by his father. The prospect of facilitating the use of custom AMT was daunting for the elder Fitzpatrick. So we involved him in the participatory design process to help understand his requirements as a carer.

During DMNI workshops, access music tutor Daniel Todd facilitates the use of music technology. Although Todd is an adept music technologist, he does not have experience in software development or electronics. We needed to build enough flexibility into the device to ensure that the instrument could also meet Todd's requirements, without the need to refactor code. At the same time, we attempted to shield Peter Fitzpatrick from the relative complexity of the functions and features Todd required. Consideration of the broader context of use resulted in the inclusion of both Todd and Peter Fitzpatrick as additional humans in the HAAT model. Therefore, we conducted user experience tests with both of them to improve usability and created user stories and use cases to capture their goals and requirements.

\section{Development}

At first glance, commercial AMT can seem expensive, perhaps understandably as niche products cannot benefit from cost reductions gained through economies of scale. At the outset of this study, it seemed as though a Raspberry Pi Zero, priced at a mere $£ 4.50$, could provide the functionality both Fitzpatricks and Todd required. In reality, technical design decisions made by the first au- thor, intended to expedite the development process, placed additional demands on the hardware. Highlevel open-source Python libraries were adopted to gain access to Bluetooth, audio, and general-purpose I/O functionality. Ultimately, an RPi3 Model A+, priced at £35, provided enough processing power to use these libraries simultaneously with acceptable latency. Although it was not in itself a significant increase in cost, this was the beginning of spiraling expenses. To meet use case requirements, off-theshelf breakout boards provided high-quality audio output and amplification, Adafruit's UDA1334A (£7) and MAX98306 (£9) boards, respectively (several products from Adafruit are used in the course of this project, further details at the company website http://adafruit.com). A Teensy LC microcontroller board (£11, for details on this and other Teensy boards used in this project, see the manufacturer website http://pirc.com) scanned the user interface and displayed information on an Adafruit 1.3-in monochrome OLED screen (£19).

The IMUs used during the prototyping stage proved to be quite unreliable. A great deal of development time was lost troubleshooting. These were unbranded boards, that seemingly utilized an InvenSense MPU-6050 (https://invensense.tdk.com) and are readily available from online retailers. At $£ 4$ each, they are remarkably cheap. After some consideration, the first author began to suspect that these boards were using a counterfeit sensor. An Adafruit BMO055 (£33) was a sophisticated, albeit more costly, replacement. When coupled with an Adafruit Feather M0 Bluefruit LE microcontroller board (£28), implementation of the wireless wristband was uncomplicated.

The development stage was expedited further by using an off-the-shelf Smoothie access switch (priced in the UK at $£ 25$, for manufacturer details see http://attainmentcompany.com/ plus Manfrotto camera lighting arms and clamps, repurposed to mount the access switch and the base unit of i1 (two units at $£ 49$ each; for further information see http://manfrotto.com). Although cost can be reduced developing such items from scratch, there are time implications in this approach. We were keen not to rush the development stage of the study as in doing so there was a danger of accruing 
unnecessary technical debt. It took approximately six months to develop il, four months longer than initially planned. Ultimately there was a relationship between ease of development and material cost, the latter totaling $£ 330$, accounting all materials. As a result of the development process, the first author realized that although Fitzpatrick's interaction with il is custom, many of the underlying technologies are not. The breakout boards, preexisting open-source code libraries, and newly developed code under-the-hood of il are likely to be useful in other custom AMT devices.

\section{Usage}

A demonstration, given before handing over the instrument, provided the two Fitzpatricks and Todd with an overview of il's features and functionality, providing an opportunity for questions. We produced a short manual, covering basic and advanced operation. As the study progressed, Peter Fitzpatrick needed further support to understand certain functions, such as how to play backing tracks, suggesting that some usability issues remained to be reconfigured and rethought. Todd demonstrated a higher degree of confidence in the facilitation of il. He struggled with implementation quirks, however, such as the file format and folder hierarchy requirements of the USB memory stick, used for backing tracks. Over time, Peter Fitzpatrick's confidence and abilities grew, resulting in him becoming the expert facilitator in the home environment. But when the elder Fitzpatrick was not available, his son was not able to use il; other carers did not possess the requisite knowledge to facilitate its use.

The younger Fitzpatrick consistently used il at least twice a week at home, during the majority of weekly DMNI workshops, and at one performance in front of an audience. Multitrack recording techniques were used in DMNI workshops to capture solo phrases, often played over ostinati or short chord progressions. Fitzpatrick would practice his improvisation techniques at home over original DMNI recordings. During the two-month period in which Fitzpatrick used the instrument, both the first author and Todd noticed, albeit anecdotally, improved control in Fitzpatrick's arm and head movements.

We had expected to perform maintenance on il during its use: fixing bugs, hardware faults, or adding features. Moreover, a failure to perform maintenance would potentially have a detrimental effect on the life span of i1. Interestingly, neither of the Fitzpatricks nor Todd reported or complained of any issues. The first author recorded one hardware fault - a loose screw-and a single software bug that caused an error in parameter values displayed. Todd suggested one extra feature: being able to adjust the release time of the guitar's amplitude envelope.

Through observation, new use cases occurred to the first author. For instance, on some days, Fitzpatrick's arm movements could be more restricted. Providing facilitators with a way to easily adjust the range of movement required to trigger the six guitar strings would add useful flexibility. It is unclear at this stage if maintenance requests would originate from the Fitzpatricks or from Todd if using the instrument independently without regular contact with the first author. Despite the small number of new feature ideas, due to time constraints, the feature set could not be expanded during this phase of the study. Altogether, Fitzpatrick enjoyed using his instrument during these two months and intends to continue using it in the future.

\section{Dependency Model of Accessing Music through Technology}

Through the development of Fitzpatrick's instrument, we realized that there were dependencies that made access to music through technology a reality for him. These were cost and resources, knowledge and skill, and time as depicted in Figure 5. Such dependencies are interconnected. For example, access to ample off-the-shelf resources can result in access barriers being lifted quickly for an individual. Although these three dependencies are somewhat pervasive in the development of new music technology, it is essential to draw attention to them in the context of inclusive music. In doing so, avoiding 
Figure 5. The dependency

model of accessing music

through technology

represents a series of

trade-offs. A shortage of

one factor can be

compensated by the

presence of another.

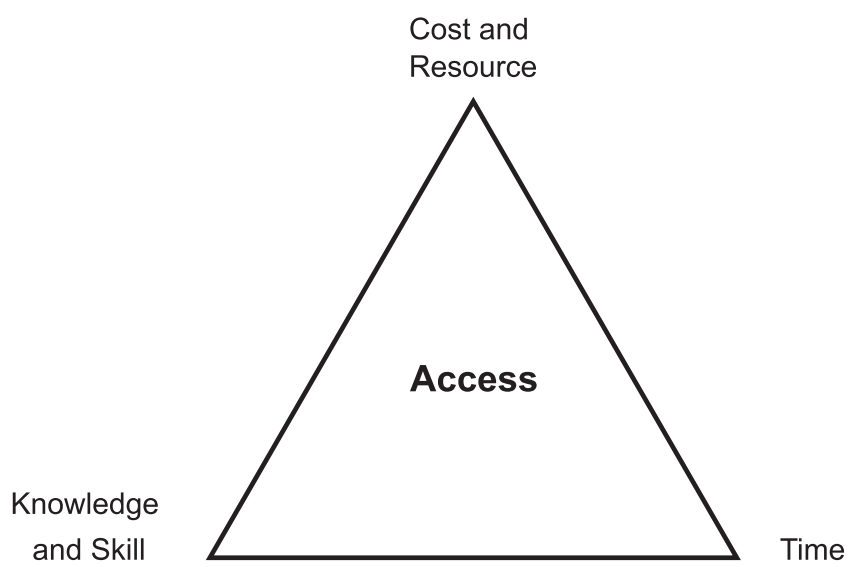

assumptions that as low-cost maker technology has the potential to remove access barriers, this will naturally happen as a matter of course.

\section{Cost and Resource Dependencies}

To use technology for removing an access barrier, such a resource needs to be available to the person facing the barrier, or there must be the means to acquire it. Fitzpatrick gained access to custom AMT through the case study described above. It is essential for academics involved in similar studies to consider how a participant might gain long-term access to an enabling device outside the confines of a study. Fitzpatrick has regular access to a commercial AMT device through DMNI workshops. Soundbeam is one of the most prominent commercial AMT devices. At an entry price of $£ 2500$, Soundbeam is likely beyond the financial means of many disabled people in the UK (DWP 2014). Financial barriers may result in relatively few disabled people owning AMT devices, using these devices at home, practicing, and gaining proficiency in their technique, in a similar way that a player of a traditional musical instrument would.

\section{Knowledge and Skill Dependencies}

A degree of specialist knowledge and skill is required to utilize music technology to remove access barriers. In Fitzpatrick's case study, knowledge and skill dependencies existed at three tiers: in facilitation, technical development, and in its use by Fitzpatrick. Facilitators may not necessarily possess the technical knowledge and skill required to develop and maintain a custom AMT. Facilitators will, however, likely have a strong understanding of the artists with whom they work, their goals, and the access barriers they face in musicking. It is vital for all stakeholders involved in the use of custom AMT to share knowledge through participation in the design process. In this study, continuous communication between Fitzpatrick, facilitators of the instrument, and the technical development team was essential for knowledge sharing and for improving his instrument.

\section{Time Dependencies}

On a practical level, it takes time to develop technology: time to write code and time to design circuits. Also, it takes time for developers to familiarize themselves with the artist and understand the requirements they might have of custom AMT. For facilitators, ease of use was of crucial importance in i1. In the home, it takes time for carers to set up and enable the use of the custom AMT. It takes time for artists to familiarize themselves with new technologies and to acquire skill in their use.

\section{Community}

The Social Model of Disability seeks to avoid a paternalistic view where disabled people are framed as in need of help and requiring support from others. The reality of digital technology use in a broader context is that any user, regardless of disability, might require support from others; for instance, when faced with a usability issue or as a result of experiencing a software bug or hardware fault. Companies involved in the manufacture of music technology products and services support their users through dedicated technical support teams with maintenance tasks factored into the schedules of engineers. Just like the first author, independent 
instrument makers may struggle to find the time to work on the maintenance of custom AMT devices and support their use. There is one potential solution to this-that of collaborating with others in such projects.

One example is e-NABLE, an online community totaling some 3,300 volunteers, dedicated to the design, fabrication, and provision of low-cost 3-D-printed prosthetics. The collaborative efforts of designers, engineers, and occupational therapists have resulted in sophisticated, standardized designs, capable of accommodating the needs of individual users through customization. One e-NABLE community member estimated in 2017 that the number of e-NABLE devices distributed was approximately 800 (Parry-Hill et al. 2017).

There is an opportunity to standardize and modularize the common underlying technologies of custom AMT devices. For example, John Kelly's custom guitar, the Kellycaster (https://www. drakemusic.org/technology/instruments-projects/ the-kellycaster), uses a sophisticated and comprehensive chord dictionary, which the first author plans to implement in il. The Kellycaster chord dictionary is already modular, implemented in a separate JSON file. Kelly-also the artist performing with the instrument-can edit and maintain this file without further technical assistance. The Kellycaster chord dictionary is implemented differently than the dictionary from i1. For each root note, up to 32 chord types are defined. Six integers represent fret positions and mute states for each string. This approach supports numerous voicings for each chord, a useful feature as voicings will differ depending on fretboard position and musical context. The il has a rudimentary implementation. A Python class, featuring MIDI and SoundFont related functionality, also declares a select few chord shapes and scales, defined as semitone transposition values for each string. A root note parameter acts as a further transposition modifier. The integration of the Kellycaster chord dictionary into il is not trivial but would pay dividends by increasing the musicality of i1. With this increased palette, the chord selection interaction design of il should be revisited, however.

Reuse of high-quality modular technical resources, integrated at the start of a project, will likely improve the development ease of custom AMTs. Following a modular structure in hardware, software, and coding procedures can make it easier to fix, update, and expand DMIs (Morreale and McPherson 2017). If several people are using one particular technology, makers may be more inclined to contribute their time towards its maintenance. Initiatives such as Drake Music England (DME) already play a vital role in connecting the somewhat disparate makers and users of custom AMT, in a similar way to e-NABLE, through DME's research and development program, the DMLab. This program centers around regular meetings in London and Manchester, attended by disabled artists, facilitators, and makers. Members collaborate to discuss, design, and test new AMT devices. The Kellycaster emerged from the DMLab.

\section{Challenges}

A challenge faced by communities such as the DMLab is getting adapted instruments and AMT into regular use with artists. In the UK, a handful of routes allow an individual to explore the idea of playing and learning an instrument. One such route is through a UK-wide network of Music Education Hubs, funded by the Arts Council England, providing and supporting music education in schools. This network allows young people to try a range of instruments and potentially discover one that resonates with them. Aside from Music Production programs, Music Hubs typically focus on the teaching of traditional musical instruments. Prospective musicians with access needs will likely struggle to (1) find information on adapted and accessible instruments and (2) find a music teacher, or music service that supports the use of such instruments.

The first of these challenges is being tackled by DME through their Accessible Musical Instrument Collection (AMIC), which has received initial funding from the Arts Council England and Clothworkers Foundation (https://www.drakemusic. org/technology/accessible-musical-instrumentcollection). A further initiative is Creative United's Guide to Buying Adaptive Musical Instruments, designed to inform music retailers, music teachers 
and those interested in learning an instrument of the adapted instruments currently available (Creative United 2020). The first stage of the AMIC project is to create a centralized collection of preexisting AMT, including both custom and commercially available equipment. Individuals will be able to visit the collection, discover available options, and learn how they might remove specific access barriers to musicking. Critically, artists will be able to try equipment during their visit, something that is not possible in regular musical instrument stores. Following this initial stage, DME will look to document the build process of the custom AMT in the collection. Currently, self-building or commissioning a build is the most direct route for an artist to obtain a custom AMT device. It is DME's intention for equipment to be easily reproducible with design files shared under open source licenses. A build process can be simplified by clear documentation, although this is a design challenge in itself. As detailed in the Complexity section, if the complexity of custom AMT is managed effectively during the design and development stages, it will be easier to document a straightforward build process. Subsequently, maintenance tasks will be simplified, supporting goals of sustained use.

The latter of the two challenges is being addressed by OHMI, which ran two pilot schemes that saw the successful use of adapted instruments within the UK education system (see https://www.ohmi. org.uk/teaching-pilot-research.html). OHMI plans to extend this service UK-wide.

\section{Complexity}

Modern digital technology is inherently complex. As we will see, the management of complexity is critical in achieving the sustained use of custom AMT. Donald Norman (2011) makes a distinction between the terms complexity and complicated. The former describes the state of the world, the latter, a state of mind. Complexity does not necessarily detract from the value of technology. Indeed, in some cases, complexity is desirable and appropriate to the task at hand; consider modular synthesizers and the complexity that naturally accompanies the exposure of large numbers of sound manipulation parameters. These parameters provide sound designers with the flexibility they value and require in their creative pursuits. A complex interface such as that of a modular synthesizer may, however, be deemed complicated by a neophyte, who may experience confusion when attempting to deduce its functionality. Complexity does not solely exist in user interfaces but also in the underlying system: in code, in electronics, and in mechanical aspects of the design. Makers may also be subject to confusion-for instance, when maintaining a codebase. Confusion may arise from a lack of experience with the technology in question but can also manifest itself through poor design. Confusion can hinder the use and maintenance of technology.

Tesler's Law of Conservation of Complexity states that a degree of complexity is inherent in a process (Saffer 2010, p. 56). The amount of complexity in a system is constant. John Maeda (2006) proposes ten laws of simplicity, the first of which is "the simplest way to achieve simplicity is through thoughtful reduction." Is the reduction of features a viable way to reduce the complexity of a custom AMT device? The answer to this question is not clear cut. It is the role of the designer to thoughtfully identify critical features, and discard those that are superfluous. A common goal in the realization of certain types of custom AMT, particularly musical instruments, is low-floor, high-ceiling design. To explain further: A novice should be able to get satisfying results from a device through intuition alone. There should be ample scope for mastery, however, only acquired through deliberate practice and development of skill. This model is present in the design of specific traditional instruments, such as the piano, and is perhaps a key to device longevity. Removal of critical features may "lower the ceiling" of the design, so to speak, reducing the overall value of the device.

Conversely, the presence of unnecessary features may result in an undue increase in complexity. During a presentation by DME at the 2020 conference on NIME, launching AMIC, instrument makers were encouraged to consider simplicity with the question, posed jovially, "Does your project have four buttons too many?" Perhaps of more relevance here is the famous maxim attributed to Einstein of 
Figure 6. Schematic of MCP72831 battery charge management controller implementation of Adafruit Feather MO
Bluefruit LE. The

implementation of the

charge status LED cannot

be changed or altered by

the user.

\section{USB \& Batt Charging}

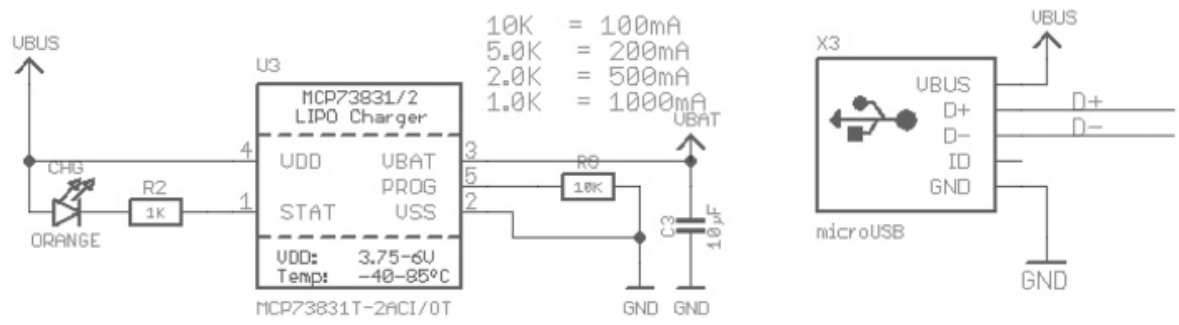

"everything should be made as simple as possible, but not simpler."

Further to his Laws of Simplicity, Madea proposes three keys. The first key is stated as "Away: More appears like less by simply moving it far, far away." Given the inherent complexity present in a custom AMT device, designers are in control of the distribution of that complexity. Complexity can be removed from a user interface, moved far away from artists and facilitators, managed by the underlying system. For example, it might be appropriate to automate certain functionality in code. There is one caveat, however: To distribute complexity effectively, the designer must have a degree of control over the fundamental elements of a system.

To reduce development complexity, a maker may resort to the use of prefabricated modular electronics or feature-rich microcontroller boards. If access to expertise is limited, a degree of system control can be lost by utilizing such technology, potentially impacting and influencing other aspects of the design. Take, for example, the wireless wristband of i1. This wristband features a lithium polymer battery, charged via USB. The user interface of the wristband gives no indication when the battery is charging or when fully charged. This lack of feedback has caused Peter Fitzpatrick some confusion, affecting the usability of il. The reason this fundamental feature is missing stems from the choice of microcontroller board used for the wristband, an Adafruit Feather MO Bluefruit LE. To make this development platform intuitive, Adafruit engineers were required to make particular design decisions. Decisions such as those surrounding the implementation of LED feedback from the MCP72831 Battery Charge Management Controller, detailed in Figure 6.

With voltage applied to the VBUS, an orange surface-mount LED provides charge-state feedback, controlled by the STAT pin. The LED flashes when charging and lights solid when charged. There is no easy way for the first author, given his skill set, to replace this LED with a panel mount equivalent, which would be visible to Peter Fitzpatrick. Although this is a relatively trivial example, it demonstrates how fundamental design decisions to manage development complexity can impact ease of use for artists and facilitators.

Poor user experience may lead to the antithesis of sustained use.

As seen in the il case study, the users of custom AMT fall into three broad categories; artists, facilitators, and makers. Users will form a mental model of a system based on a combination of external information (e.g., user documentation), and their unique past experiences, knowledge, and personal biases. Norman (2011) suggests that confusion diminishes when a user holds a lucid and correct, or at least mostly correct, mental model of the system in question. If provided, users might base their mental models on a conceptual model,that is, an abstracted representation of how a system is organized and operates (Johnson and Henderson 2002). Figure 7 is a conceptual model depicting the mapping strategies used by il. The model shows key aspects of guitar musicianship, showing correlations with the accessible interface of il.

Conceptual models do not require graphic illustration,- - they will also integrate into a device. 
Figure 7. Conceptual

model of il mapping

strategies, showing how

strumming, chord

selection, and

amplification are achieved.

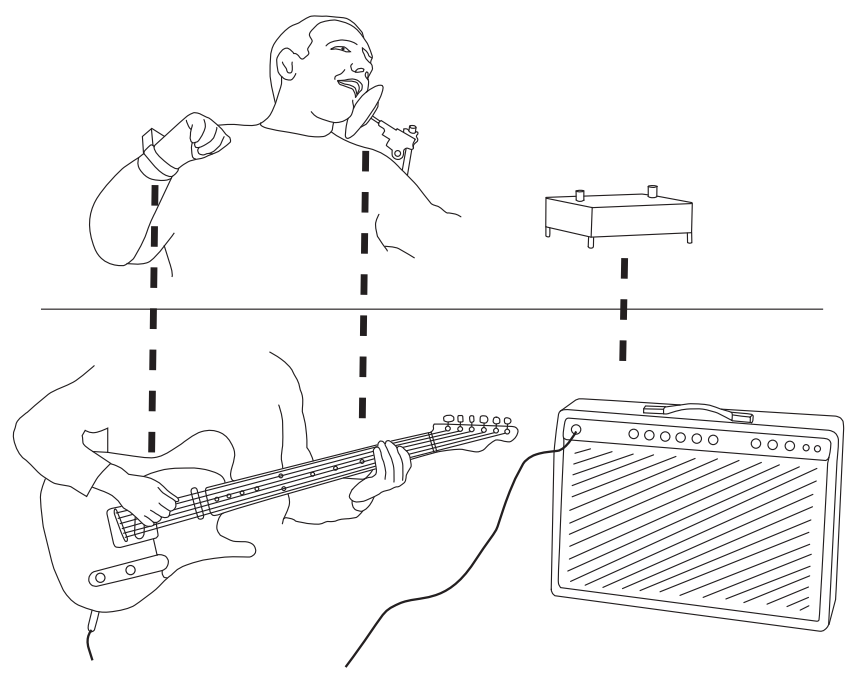

Visual signifiers, for example, the arrangement of user interface controls, can suggest the path an audio signal takes through a system. Objectoriented programming can make use of metaphors in the structuring of code. A circuit diagram can be depicted in a pseudomodular fashion, chunking functional concepts into smaller blocks of digestible information. In attending to areas such as these, the prudent designer can potentially shape the mental models of users and subsequently reduce rates of confusion.

Johnson and Henderson (2002) see conceptual models primarily as design tools, arguing for their creation at the start of the design process, following the development of user profiles and use cases but before sketching any initial interface designs. The conceptual model is fluid, of course, and indeed should be kept updated when learning more about a design through participatory design practices and technical feasibility studies. The conceptual model should focus on the task domain rather than technical implementation.

Ideally-and from an arguably utopian perspective-custom AMT should be simple to use, reproduce, maintain, and refactor. Multitiered easeof-use is a laudable goal (i.e., intuitive user interfaces with underlying systems that are straightforward to comprehend). Designing a device that accommodates usability requirements of multiple users, with different levels of technical skill, is time-consuming, however. Indeed, Johnson and Henderson note "simplicity takes time." We can, of course, forgive parsimonious practices in the development of custom AMT when time and resources are limited. Herein is where a community has a role to play.

\section{Managing Complexity as a Community}

Scozzi et al. (2008) analyzed the mental models held by community software developers working on the open-source Apache Lucene Java library. They found that the developers shared similar mental models concerning the project. Such mental models did not solely pertain to system images such as those described above. These organized knowledge structures extended to other notions-for example, team member organization, and the strategies employed by the team in software development. "Work familiarity" is a term used to describe the experience a team member has with aspects of a workplace, such as tools. If team members are familiar with the use of the same tools (e.g., the same software frameworks), it is likely they will be able to create stronger shared mental models (Espinosa et al. 2002). Espinosa and colleagues argue that distributed teams have more potential gains from shared mental models, as they do not benefit from the rich and spontaneous communications that occur with colocated ones. Robust, shared mental models are beneficial as they improve teamwork efficiency. Communication overhead reduces when domainspecific knowledge is implicit. By extension, the same observations hold for communities involved in the development of open-source hardware. DMLab is one such distributed community we can take as an example.

The work of DMLab differs from that of the Lucene Java project in that there is no singular technology that community members are collaboratively developing. Interestingly, there is a degree of work familiarity present in the DMLab community, occurring as a product of happenstance. By way of example, the Kellycaster and Jacob Harrison's Strummi (https://github.com/JacobTFH/Strummi), both use a Bela board (https://bela.io). A one-handed 
digital trombone under development at OrchLab (https://orchlab.org) and a forthcoming one-handed digital violin from our research group, Performance without Barriers, both utilize a Teensy 4.0 microcontroller and the Faust Physical Modeling Toolkit (https://faustlibraries.grame.fr/libs/physmodels). Perhaps a remit exists for DMLab to guide the community by encouraging and promoting the use of a handful of choice, underlying technologies in the development of AMT. In doing so, the community might enjoy improved efficiency in their endeavors through increased consistency in mental models held by community members.

Raspberry Pi, Bela, and Teensy 4.0 are all strong candidates for being used as a shared platform for custom AMT development within a community such as the DMLab. There are, of course, several avenues available in the development of any technology. Each route features a unique set of tradeoffs and compromises. It seems timely for the DMLab to open the discussion within the community to consider reaching a consensus on shared technologies. In addition to the efficiency benefits mentioned above, by rallying behind one particular development platform, makers will become more familiar with it. As new members join the community, they will benefit from collective knowledge and skill. This access to expertise may improve the feasibility of modifying low-level aspects of maker technologies, such as the battery charge state indicator of the Adafruit Feather M0 Bluefruit LE.

DMLab might guide the community further through a concerted effort to develop a series of conceptual models to guide the development of custom AMT, at the start of the design process, as recommended by Johnson and Henderson (2002). Such models can form a central coordination point for community members (Espinosa et al. 2002). Conceptual models of accessible digital musical instruments, in particular, can be based upon widely held cultural knowledge of traditional musical instruments. This approach features in the conceptual model of il depicted in Figure 7. Although the creation of conceptual models takes time, the shared mental models they help foster will likely make it easier for the community to repair, maintain, and refactor AMT.

\section{Summary}

We have described how custom AMTs are vital in enabling disabled artists to gain access to musicking, due to the unique way in which disability can present itself. There are dependencies at play that make the successful design, fabrication, and use of such technology a reality. There are difficulties in achieving the sustained use of custom AMTs. The HAAT model can help makers nurture an understanding of the users of custom AMT devices (i.e., artists, facilitators, and makers), and its environmental context. The provision of technical support and ongoing maintenance is required to achieve the sustained use of custom AMT. Rather than following an industry model of service-based support, makers and those involved in the use of custom AMT devices will likely benefit from being integrated into a community of practice. As Eric Raymond (2008, p 8) notes, "given enough eyeballs, all bugs are shallow." Such a community could work toward common goals through the sharing of resources, knowledge, and skill. For practical, sustained collaboration, it is vital to consider how the inherent complexity of custom AMT is managed through shared mental models and distributed efforts. Such collaborative efforts will form a participatory element of the broader musicking ecology.

\section{Acknowledgments}

We would like to thank Eoin Fitzpatrick and his team at the Drake Music Project, Northern Ireland for their continued support; the Arts and Humanities Research Council (AHRC) for funding this research; and Drake Music England for their support in writing this article.

\section{References}

Cook, A., and J. Livingston. 2010. "Need- and Task-Based Design and Evaluation." In M. Oishi et al., eds. Design and Use of Assistive Technology. Berlin: Springer, pp. 41-48. 
Creative United. 2020. "Guide to Buying Adaptive Instruments." Available online at takeitaway.org.uk/ takeitaway.org.uk/wp-content/uploads/2020/06/Guideto-Buying-Adaptive-Musical-Instruments.pdf. Accessed 19 December 2020.

Department for Transport (DfT). 2005. "Making Transport Accessible for Passengers and Pedestrians." Available online at www.gov.uk/government/publications/ inclusive-mobility. Accessed 17 November 2020.

Department for Work and Pensions (DWP). 2014. "Disability Facts and Figures." Available online at www.gov.uk/ government/publications/disability-facts-and-figures. Accessed 10 January 2020.

Espinosa, J., et al. 2002. "Shared Mental Models, Familiarity, and Coordination: A Multi-Method Study of Distributed Software Teams." In Proceedings of the International Conference on Information Systems, paper 39.

Frid, E. 2019. "Accessible Digital Musical Instruments: A Review of Musical Interfaces in Inclusive Music Practice." Multimodal Technology and Interaction 3(3):57-77.

Hitchcock, D., et al. 2006."Inclusive Mobility." Department for Transport, Mobility and Inclusion Unit. Available online at www.gov.uk/government/publications/ inclusive-mobility/inclusive-mobility\#basic-humanfactors-information. Accessed May 2021.

Johnson, J., and A. Henderson. 2002. "Conceptual Models: Begin by Designing What to Design." Interactions 9(1):25-32.

Ladner, R. 2010. "Accessible Technology and Models of Technology." In M. Oishi et al., eds. Design and Use of Assistive Technology. Berlin: Springer, pp. 25-31.

Lubet, A. 2011. Music, Disability, and Society. Philadelphia, Pennsylvania: Temple University Press.

Maeda, J. 2006. The Laws of Simplicity. Cambridge, Massachusetts: MIT Press.

Morreale, F., and A. McPherson. 2017. "Design for Longevity: Ongoing Use of Instruments from NIME 2010-2014." In Proceedings of the International Conference on New Interfaces for Musical Expression, pp. 192-197.

National Health Service (NHS). 2020. "Cerebral Palsy." Available online at www.nhs.uk/conditions/ cerebral-palsy. Accessed 10 January 2020.

Norman, D. 2011. Living with Complexity. Cambridge, Massachusetts: MIT Press.
Parry-Hill, J., et al. 2017. "Understanding Volunteer AT Fabricators: Opportunities and Challenges in DIY-AT for Others in e-NABLE." In Proceedings of the CHI Conference on Human Factors in Computing Systems, pp. 6184-6194.

Phillips, B., and H. Zhao. 1993, "Predictors of Assistive Technology Abandonment." Assistive Technology 5(1):36-45.

Phoenix, R. 2020. DIY as Privilege. London: Rough Trade Books.

Ratto, M. 2011. "Critical Making: Conceptual and Material Studies in Technology and Social Life." Information Society 27(4):252-260.

Raymond, E. 2008. The Cathedral and the Bazaar: Musings on Linux and Open Source by an Accidental Revolutionary. Sebastopol, California: O’Reilly Media.

Saffer, D. 2010. Designing for Interaction: Creating Innovative Applications and Devices. Berkeley, California: New Riders.

Samuels, K. 2016. "Enabling Creativity: A Study of Inclusive Music Technology and Practices at the Drake Music Project, Northern Ireland." PhD dissertation, Belfast, UK, Queen's University Belfast, School of Creative Arts.

Samuels, K., and F. Schroeder. 2019. "Performance without Barriers: Improvising with Inclusive and Accessible Digital Musical Instruments." Contemporary Music Review 38(5):476-489.

Scozzi, B., et al. 2008. "Shared Mental Models among Open Source Software Developers." In Proceedings of the Hawaii International International Conference on Systems Science, pp. 306-315.

Small, C. 1998. Musicking: The Meanings of Performing and Listening. Middletown, Connecticut: Wesleyan University Press.

Tanaka, A., and A. Parkinson. 2016. "Haptic Wave: A Cross-Modal Interface for Visually Impaired Audio Producers" In Proceedings of the CHI Conference on Human Factors in Computing Systems, pp. 2150-2161.

United Nations Scientific, Educational, and Cultural Organisation (UNESCO). 2018. "Education and Disability: Analysis of Data from 49 Countries." Available online at uis.unesco.org/sites/default/files/documents/ip49education-disability-2018-en.pdf. Accessed 25 April 2020.

Withers, A. 2012. Disability Politics and Theory. Black Point, Nova Scotia: Fernwood. 